\title{
Optimizing Patient Care in Egg Allergy Diagnosis and Treatment
}

\author{
Aikaterini Anagnostou ${ }^{1,2}$ \\ 'Baylor College of Medicine, Section of \\ Pediatric Immunology, Allergy and \\ Retrovirology, Houston, TX, USA; ${ }^{2}$ Texas \\ Children's Hospital, Department of \\ Pediatrics, Section of Immunology, \\ Allergy and Retrovirology, Houston, \\ TX, USA
}

\begin{abstract}
Egg allergy occurs frequently in childhood with a reported prevalence of $1.3-1.6 \%$. Providing optimal care to egg-allergic patients requires knowledge of the most up-to-date developments in both diagnosis and management, as well as effective communication skills, which will engage the patient in the shared decision-making process. This review aims to provide up-to-date information on egg allergy and also serve as a concise guide on optimal patient diagnosis and management. The field of food allergy has seen multiple advances in recent years, including use of component resolved diagnostics, early egg introduction into the infant diet as a way of preventing egg allergy, baked egg introduction and oral immunotherapy as a form of active therapy. Faced with a variety of options and treatment paths, it is important to ensure that patients and families taking part in the decision-making process have fully understood the potential outcomes and trade-offs and can undertake a detailed discussion of all options that are available to them. Shared decision-making remains the cornerstone of optimal patient care.
\end{abstract}

Keywords: food allergy, children, egg, components, prevention, food challenge, food allergy action plan, management, oral immunotherapy, desensitization, shared decision-making

\section{Introduction}

Food allergies are common, affecting $6-8 \%$ of the children ${ }^{1,2}$ and affect the quality of life of children and their caregivers. ${ }^{3}$ Food-allergic patients often worry about unintentional exposures and their consequences, especially anaphylaxis and life-threatening events. Patients face multiple dietary and psychosocial restrictions (such as exclusion from peer social activities and bullying). ${ }^{4-7}$

Optimal care for food allergy should place the best interests of the patient in its core and include the ability to adhere to evidence-based clinical guidelines, support patient needs, have an established referral system within a defined provider network and implement shared decision-making. Providing optimal care to egg-allergic patients requires knowledge of the most up-to-date developments in both diagnosis and management, as well as effective communication skills, which will engage the patient and their family in the shared decision-making process.

We live in an era when patients have access to a multitude of medical information sources, are encouraged to make their own decisions about their care and opt for the options that best fit their needs. This review aims to provide up-to-date information on egg allergy and also serve as a concise guide on optimal patient diagnosis and management.
Correspondence: Aikaterini Anagnostou Baylor College of Medicine, Section of Pediatric Immunology, Allergy and

Retrovirology, 1102 Bates Avenue Ste 330, Houston, TX, 77030, USA

Tel + I 832-824- 1319

Fax +1 832-825- 1260

Email Aikaterini.Anagnostou@bcm.edu 


\section{Epidemiology and Natural History}

Egg allergy is one of the most frequent childhood food allergies with a reported prevalence of $1.3-1.6 \% .{ }^{8,9} \mathrm{In}$ a US-based study, using data from the National Health and Nutrition Examination Survey, prevalence estimates for sensitization to egg were $3.4 \%$ for children 6 years old and over, and $14.2 \%$ for children below the age of 6 years. ${ }^{10}$ The clinical egg allergy prevalence was overestimated, but the study provided some insight into prevalence rates. ${ }^{10}$ In a recent Australian study, egg allergy was reported as affecting $8.9 \%$ of 1 -year old infants ${ }^{11}$ all of whom underwent an oral food challenge for confirmation of the diagnosis.

Egg allergy usually develops in the first year of life ${ }^{12}$ and is outgrown by the majority of patients. Knowing the natural history of egg allergy is important for the physician, in order to plan next steps in management, such as avoidance of egg or consideration of baked egg introduction (less allergenic form than cooked or raw egg) or even reintroduction of egg in all forms into the diet if the allergy has resolved. Research studies have examined the natural history of egg allergy ${ }^{13-16}$ showing that although most children will outgrow it, resolution occurs gradually, over many years. A UK prospective study in 95 egg-allergic children reported that nearly onethird had outgrown their allergy to well-cooked egg at 3 years of age and two-thirds at 6 years. ${ }^{15}$ Generally, allergy to wellcooked egg was shown to resolve twice as quickly as allergy to the uncooked form. ${ }^{15}$ In 881 patients with egg allergy in the United States, it was shown that $4 \%$ outgrew their allergy by age 4 years, $12 \%$ by age 6 years, $37 \%$ by age 10 years, and $68 \%$ by age 16 years. ${ }^{13}$ Another study examining egg allergy resolution in 213 egg-allergic children recruited from primary care, the median age of resolution was 6 years with $50 \%$ of children outgrowing their allergy by this time. ${ }^{16}$ These studies highlight geographical differences as well as population differences in the observed natural history of egg allergy. High egg-white specific IgE levels and sensitization to the stable egg-white allergen component Gal $\mathrm{d} 1$ have also been associated with persistent egg allergy. ${ }^{17}$

It is important to inform patients and families of these resolution rates, in order to address any misconceptions (some families may believe egg allergy is lifelong, for instance) and manage expectations.

\section{Diagnosis}

The gold standard diagnostic tool for egg allergy remains an oral food challenge under medical supervision. In daily practice, a clinical history of an allergic reaction occurring after ingestion of egg, together with positive testing (egg skin prick test or egg-specific IgE) is usually used for diagnosis. ${ }^{18}$ Sampson et al reported on the $95 \%$ eggspecific IgE-positive predictive value for diagnosis as 6 $\mathrm{kUA} / \mathrm{L}$, assisting clinicians in the diagnostic process. ${ }^{19}$ Their retrospective analysis included 100 with atopic dermatitis and food allergy, between 3 months and 14 years of age. ${ }^{19}$ Using the above cut-offs, physicians may also plan oral food challenges to egg accordingly.

As mentioned above, egg allergy is naturally outgrown over time by the majority of children. ${ }^{13}$ Monitoring eggspecific IgE antibodies regularly may help to decide when an egg challenge can be repeated. ${ }^{20,21}$ Both the patient and their family stand to benefit from re-introducing egg into the diet, in terms of nutrition and a wider variety of food product choices. $^{22,23}$ In addition to diagnostic cut-offs, studies have examined cut-offs for recommending an egg challenge to evaluate allergy resolution. When the chance of passing a challenge is $50 \%$ or more, most allergists would recommend an oral challenge. ${ }^{24}$ Matsui et al proposed a cutoff level for egg-specific $\operatorname{IgE}$ of $2 \mathrm{kUA} / \mathrm{L}$; almost two-thirds of patients at less than this level were able to pass their challenge. ${ }^{24}$ However, It is important to note that even when levels of egg-specific IgE are undetectable, it is still possible for patients to fail an egg challenge. ${ }^{24}$ Therefore, the decision to undertake a challenge should be made jointly by physician and patient, as part of the shared decision-making process and discussions.

There are currently no universally accepted cut-offs for either skin prick test or specific IgE that may predict which children will be able to tolerate the baked form of egg. However, component resolved diagnostics (CRDs) have emerged as a novel diagnostic tool, with improved specificity compared to traditional specific IgE testing, as well as the potential to differentiate clinical allergy from sensitization. ${ }^{25}$ In egg-allergic children, two components are used to help physicians differentiate between transient and persistent egg allergy. ${ }^{26-29}$ First, Gal d 1 (ovomucoid), a dominant allergen, which is stable against heat and digestion has been associated with persistent egg allergy and a decreased probability of cooked/heated egg tolerance. ${ }^{26-28}$ Second, Gal d 2 (ovalbumin), representing $55 \%$ of the total egg white protein, has been associated with transient egg allergy and an increased probability of cooked/heated egg tolerance. ${ }^{17,28,29}$ Identifying different 
patient phenotypes of egg allergy is an important contribution of component resolved diagnostics.

In a hypothetical scenario of an egg-allergic patient requesting diagnostic confirmation or further management options (such as baked egg introduction), CRDs and oral food challenges have a role to play in optimizing both diagnosis and care and the patient should be informed of these options, if available to them.

\section{Prevention with Early Introduction of Egg}

The timing of food allergen introduction to infants likely plays a role in the development of food allergy later in life. For many years, patients were advised to delay the introduction of allergenic foods, including egg, but this advice was reversed in recent guidelines, which strongly encourage early introduction of all common food allergens into the infant diet from 6 months of age. ${ }^{30}$ This recommendation came as a result of multiple research studies supporting the notion that early introduction of allergenic foods into an infant's diet may prevent the development of a food allergy. ${ }^{31-34}$

Specifically for egg, in the EAT study, there was a significant decrease in egg allergy for infants introducing egg at age 3 months compared with infants that were exclusively breast fed when per protocol analysis was applied (1.4 vs $5.5 \%, p=0.009) .{ }^{35}$ In an Australian study of high-risk infants with moderate to severe eczema, approximately a third of infants in the early introduction group (from 4 months of age) developed egg allergy, compared with half the infants in the late egg introduction group (from 8 months of age), $\mathrm{p}=0.11$; the assessment was performed at 1 year of age. Interestingly, even as early as 4 months of age, over one-third of the included infants already had detectable egg-specific IgE levels, despite not having any oral egg exposure. This finding suggests that sensitization to egg occurs early in life, before 4 months. ${ }^{36}$ In a larger cohort of healthy high-risk infants randomized to either raw egg or placebo from 4 to 6 months of age results were similar between the groups. Egg allergy developed in 7\% of infants receiving raw egg compared with $10.3 \%$ of infants in the placebo arm $(\mathrm{p}=$ $0.20) .{ }^{37} \mathrm{~A}$ different RCT reported a significant difference in the proportion of infants sensitized to egg at 12 months $20 \%$ versus $11 \%$ in infants randomized to placebo and egg, respectively $(\mathrm{p}=0.03){ }^{38}$
A more recent two-center Japanese study (the 'PETIT' study) ${ }^{39}$ found that the introduction of heated egg in small, gradual dose increases was both safe and effective in the prevention of egg allergy $(p=0.0001)$. A total of 147 infants were randomly assigned to early introduction of egg or placebo. Egg allergy was diagnosed in only $8 \%$ of the infants receiving $50 \mathrm{mg}$ of heated egg powder daily from 6 to 9 months of age and $250 \mathrm{mg}$ daily thereafter until 12 months of age. In the placebo group, egg allergy was diagnosed in $38 \%$ ( $p=0.0001)$. Finally, in a cross-sectional study by Koplin et al, which included 2589 infants, a lower rate of egg allergy was noted for participants who received cooked egg at 4-6 months than those who received cooked egg after that age. ${ }^{40}$ Older age of introducing egg increased the risk of developing egg allergy in both low-risk infants and high-risk infants (those with eczema, history of reactions to foods, or a family history of food allergy) with reported $\mathrm{p}=0.22$ and $\mathrm{p}<0.001$, respectively. ${ }^{40}$

In addition to the above research studies, a recent systematic review and meta-analysis, which included six RCTs and a total of 3032 participants, reported a protective effect with early egg introduction with 37 fewer cases of egg allergy per 1000 people (assuming a 9.3\% incidence of egg allergy in the studied population). ${ }^{41}$

Recent Australian data show a complete change in the behavior of new parents with earlier introduction of egg in their infants' diet following publication and dissemination of the new guidelines supporting early introduction. ${ }^{42}$ The majority of parents fed their infants cooked (rather than baked) egg in an effort to limit the amount of sugar in the diet. It is reported that the majority of Australian parents are following the new recommendations from the government and medical societies and are successfully feeding their infants egg by 1 year of age, which is much earlier than what was seen previously. ${ }^{42}$

\section{Management}

The optimal management of egg allergy includes various components, such as medical interventions, dietetic input and active treatment approaches, such as introduction of baked egg and oral immunotherapy. These components are explored in more detail below.

\section{Medical Management}

A comprehensive management plan or "Food allergy Action plan" is essential and should include advice on identification and treatment of allergic reactions as well as education on the use of epinephrine auto-injectors. ${ }^{43,44}$ The management 
plan needs to include not only immediate family members but also members of the wider environment of the child, such as nannies, grandparents and school personnel. Reactions to accidental exposures may occur, but education on how to quickly identify the relevant signs and symptoms and how to promptly administer the appropriate treatment are key to mitigate negative outcomes. ${ }^{44,45}$

Provision of emergency medication is another key element of medical management. Epinephrine is the first-line treatment for anaphylaxis, and long-acting oral antihistamines may be used for mild reactions. ${ }^{44,46}$

Finally, patient follow-up by a qualified allergist is important. The allergist may provide ongoing education on preventing and managing future reactions as well as perform allergy tests periodically to assess for natural resolution. Additionally, for patients who are avoiding all forms of egg, regular assessments should be made to evaluate for baked egg challenges and introduction.

\section{Dietary Management}

All patients require clear information on egg avoidance. ${ }^{47}$ Dietitians play a key role in educating patients and families in this area. ${ }^{48}$ Education should include discussions on food labelling (both ingredients list and "may contain" statements) and patients should be advised to read both the ingredients list and the precautionary allergen labelling (PAL) on the foods they buy, every time, as recipes may change over time. ${ }^{49}$ Patients often find PAL statements difficult to interpret without dietetic input. When eating outside the home patients need to be educated on certain risks. Miscommunication is a common problem, so patients should liaise directly with staff in restaurants and other food establishments to avoid egg "contamination" (also known as "cross contact") in their meal. ${ }^{50}$ Clear communication (such as alerting the restaurant in ahead of time of the food allergy, involving senior staff or the chef in the order, and ensuring the table surface is adequately cleaned) is key in minimizing risk. ${ }^{51}$ Cross-contact is common in buffets where food is shared and cleaning tends not to be thorough. Parents should be advised that simple dishes with ingredients that are clearly stated are often a better option than complex dishes with multiple different ingredients and complicated recipes. $^{50}$

\section{Role of Baked Egg Introduction}

Different forms of egg display different allergenicity, with raw egg being the most allergenic and baked egg the least.
Generally, heating appears to reduce allergenicity. ${ }^{52}$ Studies suggest that up to $70 \%$ of egg-allergic children are able to tolerate egg that is baked. ${ }^{53}$ This provides some benefits such as a more inclusive diet, better nutrition, less social restrictions and, as a result, better quality of life.

The role of baked egg introduction in the resolution of egg allergy is more controversial, with a recent systematic review suggesting that more evidence is needed in this area, especially in the form of randomized trials of baked egg consumption versus baked egg avoidance in bakedtolerant participants. ${ }^{54}$ However, multiple observational research studies report a beneficial effect with regular baked egg consumption. ${ }^{55,56}$ Children who react to baked egg in their initial exposure appear to have a more severe phenotype and lower rates of natural resolution. In contrast, children with negative or low sensitization to Gal d 1 are more likely to tolerate baked/heated egg. ${ }^{26,27}$ This information may be helpful to the clinician when considering an oral challenge and introduction of baked egg.

Peters et al examined 140 infants with challengeconfirmed egg allergy at age 1 year and reported egg allergy resolution in 47\%, 12 months later (at age 2). ${ }^{55}$ Those infants who had reacted to baked egg at baseline, showed lower rates of resolution. Additionally, infants that consumed baked egg more than 5 times per month had better odds in developing tolerance. ${ }^{55}$ A similar study, which included 70 participants who regularly ate baked egg, evaluated the role of baked egg in the development of tolerance to regular egg. ${ }^{56}$ A reported $53 \%$ of the above participants were able to tolerate regular egg after an approximately 3-year study period, compared with only $28 \%$ of the participants in the comparison group (who all adhered to strict avoidance). Additionally, regular baked egg consumers were 14 times more likely to develop tolerance compared with the strict avoidance group and tolerance was achieved much earlier (50 months versus 78.7 months in the avoidance group). ${ }^{56}$

As part of optimal care, the role of baked egg introduction should be discussed with patients, including benefits (nutritional, social) and risks (allergic reactions during the challenge), as this simple intervention has the potential to change the daily life of the egg-allergic child and potentially speed up their allergy resolution.

\section{Egg Oral Immunotherapy}

Food oral immunotherapy (OIT) is a new form of active therapy for food allergies. The administration of small but 
increasing doses of the allergenic food allows patients to gradually increase their ability to consume small to medium amounts of their allergen without reactions. ${ }^{57}$ Food oral immunotherapy studies have been conducted for most of the common childhood food allergens, including egg. ${ }^{58-61}$

In a seminal randomized oral immunotherapy study of 55 children, with egg allergy, a 55\% rate of desensitization was noted in the active arm after 10 months of therapy compared with $0 \%$ in the placebo arm. An additional year of egg OIT increased the desensitization rate to $75 \%$ supporting the notion that longer treatment periods likely result in higher desensitization rates ${ }^{59}$. A 4-year follow-up of the above subjects has reinforced the above statement showing that longer duration of therapy increases the likelihood of tolerating regular egg. ${ }^{58}$

A recent trial evaluated 50 participants aged 3-16 years, which were reactive to unbaked egg and were assigned to 2 years of treatment with either baked egg or egg OIT. ${ }^{62}$ A comparison group of baked egg-reactive children were also included and received egg OIT. Following 2 years of treatment, therapy was discontinued for $8-10$ weeks to examine sustained unresponsiveness. ${ }^{62}$ A total of $11.1 \%$ of children consuming baked egg compared with $43.5 \%$ of children receiving egg OIT achieved the desired outcome (sustained unresponsiveness, $\mathrm{P}=0.009$ ).$^{62}$ In the baked eggreactive group, $17.9 \%$ achieved sustained unresponsiveness. Children receiving egg OIT were much more likely to reach the desired outcome compared with those consuming baked egg alone (without OIT). ${ }^{62}$

Finally, a systematic review investigating efficacy and safety of egg OIT included 10 RCTs and a total of 439 participants between 1 and 18 years. ${ }^{63}$ The majority of children receiving egg OIT were able to consume a partial serving of egg (1-7.5 g) compared to only $10 \%$ in the avoidance group. A full portion of egg was successfully consumed by almost $50 \%$ in the OIT group compared to $10 \%$ of the avoidance group. ${ }^{63}$ The authors reported that OIT induces tolerance in almost $50 \%$ of the subjects compared with only 1 in 10 subjects who follow egg avoidance. ${ }^{63}$ However, adverse events are much more frequent in the treatment arm and some children (reported as 1 in 12 study participants) may suffer severe allergic reactions requiring epinephrine. ${ }^{63}$

It appears that oral immunotherapy for egg allergy is effective, but this is not without risk. The risk-benefit ratio should be clearly outlined and discussed in detail with patients and families, so they can make the decision that best fits their expectations and preferences.

\section{Shared Decision-Making}

Shared decision-making is important for any disease with long-term management, such as egg allergy. ${ }^{64,65}$ As there are multiple different management and therapy options available to patients currently, each with its own benefits and risks, shared decision-making presents significant value. ${ }^{66}$

The landscape of egg allergy has evolved in both diagnostics and treatment over recent years, with a variety of novel interventions such as component resolved diagnostics, oral immunotherapy, and early introduction with the aim to prevent egg allergy development. This rapid advancement can be challenging to patients as they try to understand potential outcomes and trade-offs of different available options, and decide which aspects of management matter to them the most, so they can reach a decision on how to proceed. ${ }^{66,67}$ See Figure 1.

The role of young children in decision-making discussions should also be emphasized and youngsters should be encouraged to participate in decisions about their care, if they wish, from an appropriate age. Both parents and healthcare providers have a duty to support children as they navigate the - often confusing - landscape of their disease and learn to make decisions on their own. The young patient's concerns may be addressed during the

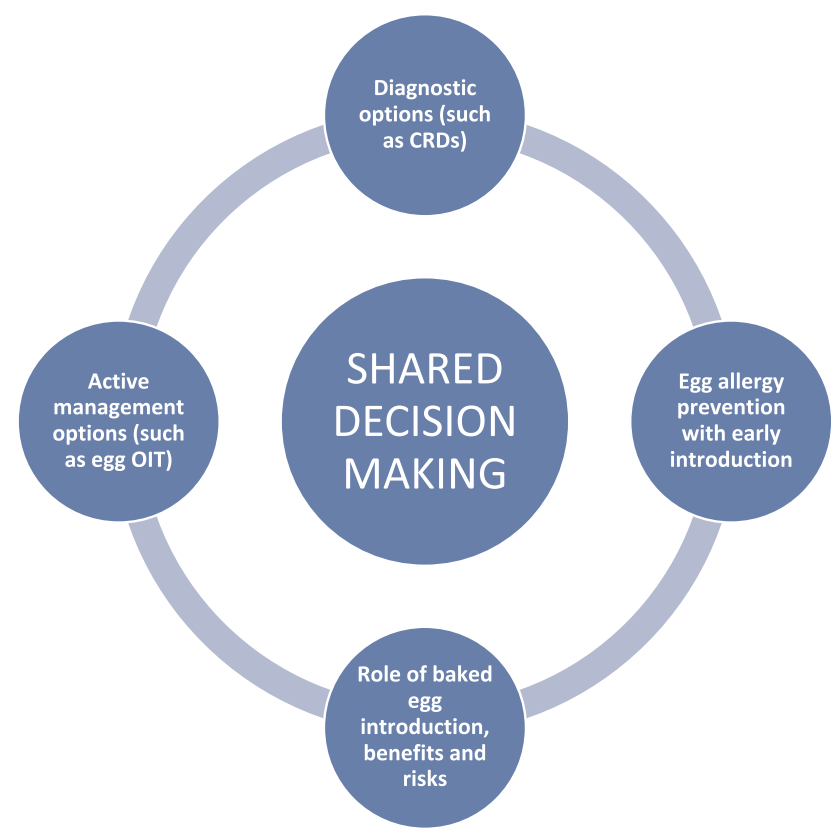

Figure I Optimizing patient care in egg allergy.

Abbreviations: CRDs, component resolved diagnostics; OIT, oral immunitherapy. 
clinic visit by the allergist, their opinions should be sought and their preferences for care should be investigated and discussed. Children may sometimes not reveal their lack of understanding of complex concepts, so a jargon-free language would be useful to assist them in this area. ${ }^{68}$ Parents may also engage their children separately in decisionmaking and ensure they are ready to transition to independence during adolescent and adult years. ${ }^{68}$

A relationship of trust and respect between patients, families and clinicians in combination with a safe, unrushed environment where different opinions may be heard and analyzed, and validated decision aids may be incorporated into patient care will provide the patient with a valuable and optimal care experience. ${ }^{69}$

\section{Conclusions}

It is clear that optimizing the care of the egg-allergic patient includes multiple elements, both in terms of diagnosis and management. These need to be addressed by the physician looking after the patient so that jointly, patients/ families and physicians may discuss available options and treatment pathways and make appropriate decisions. It is important to note that shared decision-making remains the cornerstone of optimal patient care and should be applied in all relevant interactions in the allergy clinic.

\section{Abbreviations}

OIT, oral immunotherapy; PAL, precautionary allergen labelling; QoL, quality of life; RCT, randomized controlled trials.

\section{Funding}

There is no funding to report.

\section{Disclosure}

None directly relevant to this work.

Dr Aikaterini Anagnostou is the principal investigator for Aimmune Therapeutics research peanut oral immunotherapy trials and receives Institutional grant funding, and serves as an advisory board member for DBV Technologies.

\section{References}

1. Gupta RS, Springston EE, Warrier MR, et al. The prevalence, severity, and distribution of childhood food allergy in the United States. Pediatrics. 2011;128(1):e9-17. doi:10.1542/peds.2011-0204

2. Jiang J, Warren CM, Gupta RS. Food allergy: epidemiology and racial/ ethnic differences. J Food Allergy. 2020;1:11-16.
3. Avery NJ, King RM, Knight S, Hourihane JOB. Assessment of quality of life in children with peanut allergy. Pediatr Allergy Immunol. 2003;14:378-382. doi:10.1034/j.1399-3038.2003.00072.x

4. Stensgaard A, Nielsen D, Munch M, Dunngalvin A. Quality of life in childhood, adolescence and adult food allergy: patient and parent perspectives. Clin Exp Allergy. 2016;530-539. doi:10.1111/cea.12849

5. Greenhawt M. Food Allergy Quality of Life. Ann Allergy, Asthma Immunol. 2016;113:506-512. doi:10.1016/j.anai.2014.06.027

6. Herbert L, Shemesh E, Bender B. Clinical management of psychosocial concerns related to food allergy. J Allergy Clin Immunol Pract. 2016;4:205-213. doi:10.1016/j.jaip.2015.10.016

7. Annunziato RA, Rubes M, Ambrose MA, et al. Longitudinal evaluation of food allergy-related bullying. J Allergy Clin Immunol Pract. 2014;2:639-641. doi:10.1016/j.jaip.2014.05.001

8. Eggesbø M, Botten G, Halvorsen R, Magnus P. The prevalence of allergy to egg: a population-based study in young children. Allergy. 2001;56:403-411. doi:10.1034/j.1398-9995.2001.056005403.x

9. Nickel R. Sensitization to hen's egg at the age of twelve months is predictive for allergic sensitization to common indoor and outdoor allergens at the age of three years. $J$ Allergy Clin Immunol. 1997;99:613-617. doi:10.1016/S0091-6749(97)70021-6

10. Salo PM, Arbes SJ, Jaramillo R, et al. Prevalence of allergic sensitization in the United States: results from the National Health and Nutrition Examination Survey (NHANES) 2005-2006. J. Allergy Clin. Immunol. 2014;134:350-359. doi:10.1016/j.jaci.2013.12.1071

11. Osborne NJ, Koplin JJ, Martin PE, et al. Prevalence of challenge-proven IgE-mediated food allergy using population-based sampling and predetermined challenge criteria in infants. J. Allergy Clin. Immunol. 2011;127:668-76.e1-2. doi:10.1016/j.jaci.2011.01.039

12. Kulig M, Bergmann R, Klettke U. Natural course of sensitization to food and inhalant allergens during the first 6 years of life. J Allergy. 1999;220-228. doi:10.1034/j.1398-9995.1999.00753.x

13. Savage JM, Skripak E, Justin Wood R, Wood RA. The natural history of egg allergy. J. Allergy Clin. Immunol. 2007;120:1413-1417. doi:10.1016/j.jaci.2007.09.040

14. Ford RP, Taylor B. Natural history of egg hypersensitivity. Arch Dis Child. 1982;57:649-652. doi:10.1136/adc.57.9.649

15. Clark A, Islam S, King Y, et al. A longitudinal study of resolution of allergy to well-cooked and uncooked egg. Clin Exp Allergy. 2011;41:706-712. doi:10.1111/j.1365-2222.2011.03697.x

16. Sicherer SH, Wood RA, Vickery BP, et al. The natural history of egg allergy in an observational cohort. J. Allergy Clin. Immunol. 2014;133:492-499. doi:10.1016/j.jaci.2013.12.1041

17. Alessandri C, Zennaro D, Scala E, et al. Ovomucoid (Gal d 1) specific IgE detected by microarray system predict tolerability to boiled hen's egg and an increased risk to progress to multiple environmental allergen sensitisation. Clin Exp Allergy. 2012;42:441-450. doi:10.1111/j.1365-2222.2011.03915.x

18. Sicherer SH, Sampson HA. Food allergy: epidemiology, pathogenesis, diagnosis, and treatment. J Allergy Clin Immunol. 2013;13 3:291-307.e5.

19. Sampson HA. Utility of food-specific IgE concentrations in predicting symptomatic food allergy. J Allergy Clin Immunol. 2001;10 7:891-896. doi:10.1067/mai.2001.114708

20. By-H T, Jo'B H. Monitoring of IgE-mediated food allergy in childhood. Acta Paediatr. 2004;93:759-764. doi:10.1080/0803525 0410029399

21. Shek LPC, Soderstrom L, Ahlstedt S, Beyer K, Sampson H. a. Determination of food specific IgE levels over time can predict the development of tolerance in cow's milk and hen's egg allergy. J Allergy Clin Immunol. 2004;114:387-391. doi:10.1016/j.jaci.2004.04.032

22. DunnGalvin A, Cullinane C, Daly DA, et al. Longitudinal validity and responsiveness of the Food Allergy Quality of Life Questionnaire - Parent Form in children 0-12 years following positive and negative food challenges. Clin Exp Allergy. 2010;40:476-485. doi:10.1111/ j.1365-2222.2010.03454.x 
23. van der Velde JL. Food allergy-related quality of life after double-blind, placebo-controlled food challenges in adults, adolescents, and children. J Allergy Clin Immunol. 2012;130:1136-1143.e2. doi:10.1016/j.jaci.2012.05.037

24. Perry TT, Matsui EC, Kay Conover-Walker M, Wood RA. The relationship of allergen-specific IgE levels and oral food challenge outcome. J Allergy Clin Immunol. 2004;114:144-149. doi:10.1016/j. jaci.2004.04.009

25. Sastre J, Sastre-Ibañez M. Molecular diagnosis and immunotherapy. Curr Opin Allergy Clin Immunol. 2016;16:565-570. doi:10.1097/ ACI.0000000000000318

26. Palosuo K, Kukkonen AK, Pelkonen AS, Mäkelä MJ. Gal d 1-specific IgE predicts allergy to heated egg in Finnish children. Pediatr Allergy Immunol. 2018;29:637-643. doi:10.11 11/pai.12954

27. Dang TD, Peters RL, Koplin JJ, et al. Egg allergen specific $\operatorname{IgE}$ diversity predicts resolution of egg allergy in the population cohort HealthNuts. Allergy Eur J Allergy Clin Immunol. 2019;74:318-326. doi:10.1111/all.13572

28. Caubet J-C, Kondo Y, Urisu A, Nowak-Węgrzyn A. Molecular diagnosis of egg allergy. Curr Opin Allergy Clin Immunol. 2011;11:210-215. doi:10.1097/ACI.0b013e3283464d1b

29. Matricardi PM. EAACI molecular allergology user's guide. Pediatr Allergy Immunol. 2016;27(Suppl 2):1-250.

30. Institute Of Allergy N, Diseases I. Addendum Guidelines for the Prevention of Peanut Allergy in the United States: summary for Clinicians. JACI.

31. Katz Y, Rajuan N, Goldberg MR, et al. Early exposure to cow's milk protein is protective against IgE-mediated cow's milk protein allergy. J. Allergy Clin. Immunol. 2010;126:77-82.e1. doi:10.1016/j.jaci.20 10.04 .020

32. Du Toit G. Early consumption of peanuts in infancy is associated with a low prevalence of peanut allergy. J Allergy Clin Immunol. 2008;122:984-991. doi:10.1016/j.jaci.2008.08.039

33. Du Toit G. Effect of avoidance on peanut allergy after early peanut consumption. $N$ Engl $J$ Med. 2016:1-9. doi:10.1056/NEJMoa 1514209 .

34. Ierodiakonou D. Timing of allergenic food introduction to the infant diet and risk of allergic or autoimmune disease a systematic review and meta-analysis. JAMA. doi:10.1001/jama.2016.12623

35. Perkin MR. Randomized trial of introduction of allergenic foods in breast-fed infants. N Engl J Med. 2016;374:1733-1743. doi:10.1056/ NEJMoa1514210

36. Palmer DJ, Metcalfe J, Makrides M, et al. Early regular egg exposure in infants with eczema: a randomized controlled trial. J. Allergy Clin. Immunol. 2013. doi:10.1016/j.jaci.2013.05.002

37. Palmer DJ, Sullivan TR, Gold MS, Prescott SL, Makrides M. Randomized controlled trial of early regular egg intake to prevent egg allergy. J. Allergy Clin. Immunol. 2017;139:1600-1607.e2. doi:10.1016/j.jaci.2016.06.052

38. Wei-Liang Tan J, Valerio C, Barnes EH, et al. A randomized trial of egg introduction from 4 months of age in infants at risk for egg allergy. J. Allergy Clin. Immunol. 2017;139:1621-1628.e8. doi:10.10 16/j.jaci.2016.08.035

39. Natsume O. Two-step egg introduction for prevention of egg allergy in high-risk infants with eczema (PETIT): a randomised, double-blind, placebo-controlled trial. Lancet. 2017;389 (10066):276-286. doi:10.1016/S0140-6736(16)31418-0

40. Koplin JJ, Osborne NJ, Wake M, et al. Can early introduction of egg prevent egg allergy in infants? A population-based study. J. Allergy Clin. Immunol. 2010;126:807-813. doi:10.1016/j.jaci.2010.07.028

41. Al-Saud B, Sigurdardóttir ST. Early introduction of egg and the development of egg allergy in children: a systematic review and meta-analysis. Int Arch Allergy Immunol. 2018;177:350-359. doi:10.1159/000492131
42. Soriano VX, Peters RL, Ponsonby A-L, et al. Earlier ingestion of peanut after changes to infant feeding guidelines: the EarlyNuts study. J. Allergy Clin. Immunol. 2019;144:1327-1335.e5. doi:10.1016/j.jaci.2019.07.032

43. Anagnostou K, Stiefel G, Brough H, et al. Active management of food allergy: an emerging concept. Arch. Dis. Child. 2015;100:386-390. doi:10.1136/archdischild-2014-306278

44. Boyce JA, Assa'ad A, Burks AW, et al. Guidelines for the diagnosis and management of food allergy in the United States: report of the NIAID-sponsored expert panel. $J$ Allergy Clin Immunol. 2010;126:1105-1118. doi:10.1016/j.jaci.2010.10.008

45. Anagnostou K. Active management of food allergy: an emerging concept. Arch Dis Child. 2014:1-5. doi:10.1136/archdischild-2014306278.

46. Anagnostou K. Anaphylaxis in Children: epidemiology, Risk Factors and Management. Curr Pediatr Rev. 2018;14:180-186. doi:10.2174/ 1573396314666180507115115

47. Kim JS, Sicherer SH. Living with food allergy: allergen avoidance. Pediatr Clin North Am. 2011;58:459-470. doi:10.1016/j.pcl.2011.02.007

48. Hubbard S. Nutrition and food allergies: the dietitian's role. Ann Allergy, Asthma Immunol. 2003;90:115-116. doi:10.1016/S10811206(10)61672-9

49. Zurzolo GA, Mathai ML, Koplin JJ, Allen KJ. Hidden allergens in foods and implications for labelling and clinical care of food allergic patients. Curr Allergy Asthma Rep. 2012;12:292-296. doi:10.1007/ s11882-012-0263-6

50. Carter CA, Pistiner M, Wang J, Sharma HP. Food allergy in restaurants work group report. $J$ Allergy Clin Immunol Pract. 2020;8:70-74. doi:10.1016/j.jaip.2019.09.013

51. Leftwich J, Barnett J, Muncer K, et al. The challenges for nut-allergic consumers of eating out. Clin Exp Allergy. 2011;41:243-249. doi:10.1111/j.1365-2222.2010.03649.x

52. Nowak-Wegrzyn A, Fiocchi A. Rare, medium, or well done? The effect of heating and food matrix on food protein allergenicity. Curr Opin Allergy Clin Immunol. 2009;9:234-237. doi:10.1097/ACI.0b0 $13 \mathrm{e} 32832 \mathrm{~b} 88 \mathrm{e} 7$

53. Lemon-Mulé H, Sampson HA, Sicherer SH, et al. Immunologic changes in children with egg allergy ingesting extensively heated egg. J. Allergy Clin. Immunol. 2008;122:977-983.e1. doi:10.1016/j. jaci.2008.09.007

54. Lambert R, Grimshaw KEC, Ellis B, Jaitly J, Roberts G. Evidence that eating baked egg or milk influences egg or milk allergy resolution: a systematic review. Clin Exp Allergy J Br Soc Allergy Clin Immunol. 2017;47:829-837. doi:10.1111/cea.12940

55. Peters RL. The natural history and clinical predictors of egg allergy in the first 2 years of life: a prospective, population-based cohort study. J Allergy Clin Immunol. 2017;133(485-491.e6).

56. Leonard SA, Sampson HA, Sicherer SH, et al. Dietary baked egg accelerates resolution of egg allergy in children. $J$ Allergy Clin Immunol. 2012;130:473-80.e1. doi:10.1016/j.jaci.2012.06.006

57. Anagnostou K. Food immunotherapy for children with food allergies: state of the art and science. Curr Opin Pediatr. 2018;30:798-805. doi:10.1097/MOP.0000000000000684

58. Jones SM, Burks AW, Keet C, et al. Long-term treatment with egg oral immunotherapy enhances sustained unresponsiveness that persists after cessation of therapy. J. Allergy Clin. Immunol. 2016;137:1117-1127.e10. doi:10.1016/j.jaci.2015.12.1316

59. Burks AW, Jones SM, Wood RA, et al. Oral immunotherapy for treatment of egg allergy in children. $N$ Engl $J$ Med. 2012;367:233-243. doi:10.1056/NEJMoa1200435

60. Buchanan AD, Green TD, Jones SM, et al. Egg oral immunotherapy in nonanaphylactic children with egg allergy. J. Allergy Clin. Immunol. 2007;119:199-205. doi:10.1016/j.jaci.2006.09.016

61. Wood RA. Food allergen immunotherapy: current status and prospects for the future. $J$ Allergy Clin Immunol. 2016;137:973-982. doi:10.1016/j.jaci.2016.01.001 
62. Kim EH, Perry TT, Wood RA, et al. Induction of sustained unresponsiveness after egg oral immunotherapy compared to baked egg therapy in children with egg allergy. J. Allergy Clin. Immunol. 2020;146:851-862.e10. doi:10.1016/j.jaci.2020.05.040

63. Romantsik O, Tosca MA, Zappettini S, Calevo MG. Oral and sublingual immunotherapy for egg allergy. Cochrane Database Syst Rev. 2018;4:CD010638. doi:10.1002/14651858.CD010638.pub3

64. Fiks A. Parent-reported outcomes of a shared decision-making portal in asthma: a practice-based RCT. Pediatrics. 2015;135:e965-e973. doi:10.1542/peds.2014-3167

65. Fiks AG, Mayne S, Karavite DJ, DeBartolo E, Grundmeier RW. A shared e-decision support portal for pediatric asthma. J Ambul Care Manage. 2014;37:120-126. doi:10.1097/JAC.0000000000000 025
66. Anagnostou A, Hourihane JOB, Greenhawt M. The role of shared decision making in pediatric food allergy management. J Allergy Clin Immunol Pract. 2019;46-51. doi:10.1016/j.jaip.2019.09.004

67. Blaiss MS, Steven GC, Bender B, et al. Shared decision making for the allergist. Ann. Allergy, Asthma Immunol. 2019;122:463-470. doi:10.1016/j.anai.2018.08.019

68. Miller VA. Involving youth with a chronic illness in decision-making: highlighting the role of providers. Pediatrics. 2018;142:S142-S148. doi:10.1542/peds.2018-0516D

69. Shaker M, Hsu Blatman K, Abrams EM. Engaging patient partners in state-of-the-art allergy care: finding balance when discussing risk. Ann Allergy, Asthma Immunol. 2020;125:252-261.

\section{Publish your work in this journal}

The Journal of Asthma and Allergy is an international, peer-reviewed open-access journal publishing original research, reports, editorials and commentaries on the following topics: Asthma; Pulmonary physiology; Asthma related clinical health; Clinical immunology and the immunological basis of disease; Pharmacological interventions and new therapies. The manuscript management system is completely online and includes a very quick and fair peer-review system, which is all easy to use. Visit http://www.dovepress.com/testimonials.php to read real quotes from published authors. 